\title{
Actualización: Infección por Virus de la Inmunodeficiencia Humana y Sindrome de Inmunodeficiencia Adquirida
}

\author{
Infection with Human Immunodeficiency Virus and Acquired Immunodeficiency Syndrome
}

Waldo H. Belloso

\begin{abstract}
Resumen
A más de tres décadas del inicio de la era del SIDA, el avance de la terapéutica sobre el control de la infección por VIH ha sucedido y sucede a una velocidad prácticamente sin precedentes en la historia de las enfermedades infecciosas. Esto ha convertido a la infección por VIH-SIDA en una entidad diferente con nuevos desafíos, modificando completamente sus paradigmas. Hoy la tarea asistencial debe incluir dentro de la agenda la inserción social, la adherencia a la medicación y la planificación a largo plazo. Es con esta intención que se han sugerido aquí algunas potenciales áreas de interacción entre el médico de atención primaria y el especialista.Sin embargo, es importante recordar que nuestra tarea médica no sólo implica una responsabilidad sobre el paciente individual (sobre la cual se ha centrado este texto) sino también sobre la sociedad. Esta es la epidemia que nos toca vivir, y cada uno debe encontrar su lugar en la lucha contra el VIH.

Abstract

More than three decades into the AIDS era, the advancement of therapeutics for the control of HIV infection has happened and is happening at a rate almost unprecedented in the history of infectious diseases. This has made HIV-AIDS in a different entity with new challenges, completely changing their paradigms. Today's health care programs should include in their agenda social inclusion, medication adherence and long-term planning. It is with this intention that have been suggested here some potential areas of interaction between the primary care physician and the specialist. However, it is important to remember that our medical task not only implies a responsibility on the individual patient (on which this text has focused) but also on society. This is the epidemic in which we live, and everyone should find their place in the fight against HIV.
\end{abstract}

Belloso WH. Infección por Virus de la Inmunodeficiencia Humana y Síndrome de Inmunodeficiencia Adquirida. Evid Act Pract Ambul. Abr-Jun 2014;17(2):68-76.

\begin{abstract}
Introducción y epidemiología
La infección por el virus de la inmunodeficiencia humana $(\mathrm{VIH})$ constituye sin dudas uno de los principales problemas globales de salud pública en la actualidad. Alrededor de 40 millones de personas se encuentran hoy infectadas por el virus en el mundo y, si bien los avances en términos de accesibilidad a los tratamientos específicos que hemos vivido en los últimos años han sido enormes, casi inimaginables hace algunos años, aún queda mucho camino por recorrer en la contención final de esta epidemia.
\end{abstract}

Hoy no cabe duda que la disponibilidad del tratamiento antirretroviral de combinación -incluyendo al menos tres fármacos de al menos dos familias- permite cambiar la historia natural de la infección por VIH tanto a nivel poblacional como para un paciente individual. Aun en el contexto de diagnósticos tardíos, es hoy mucho más raro observar severos deterioros inmunológicos progresivos y enfermedades oportunistas asociadas a inmunosupresión profunda. Por otra parte, existe un cambio epidemiológico significativo en relación a la población que es asistida por infección por VIH y SIDA en la actualidad. Tanto por la mayor supervivencia de los pacientes bajo tratamiento antirretroviral como por el incremento progresivo en la edad promedio en la cual se establecen los nuevos diagnósticos, los pacientes que asistimos con inmunodeficiencia adquirida son cada vez más añosos y por tanto presentan toda una serie de características particulares y de condiciones patológicas asociadas (comorbilidades) que condicionan tanto las manifestaciones clínicas de la infección como así también su pronóstico y también, en alguna medida, la respuesta al tratamiento.

Condiciones asociadas tales como la hipertensión arterial, la dislipidemia, la diabetes, la osteopenia, la insuficiencia renal crónica se agregan al tabaquismo, la coinfección con virus de Hepatitis B y C y otras situaciones previamente reconocidas como frecuentes en personas con infección por VIH. Pero además, el propio VIH presenta una relación con el desarrollo, la progresión o la respuesta terapéutica de varias de estas condiciones. Por lo que se establece un doble vínculo en el cual distintas comorbilidades son más frecuentes por las nuevas características epidemiológicas de la población, pero a su vez también se encuentran favorecidas por la presencia del virus.
La comprensión de este doble vínculo es fundamental para valorar la real importancia de la situación clínica del paciente a la hora de decidir el inicio del tratamiento y la combinación de fármacos que se va a utilizar para cada caso particular.

La relación entre el VIH y las enfermedades crónicas no marcadoras de SIDA fue establecida tanto en diferentes cohortes como así también en ensayos clínicos tales como el estudio SMART. En este estudio se estableció que la interrupción del tratamiento antirretroviral condicionaba un incremento del riesgo de eventos clínicos serios no marcadores de SIDA, en contra de lo que se había hipotetizado.

La relación entre la magnitud de la inmunodeficiencia y el riesgo de aparición de enfermedad cardiovascular, hepatopatías crónicas, insuficiencia renal crónica o distintos tipos de cáncer no marcador de SIDA, fue establecida y ratificada por diferentes cohortes. La mayor importancia de esta vinculación radica en que si bien el riesgo absoluto de padecer un evento clínico es menor en pacientes con mayores niveles de linfocitos $T$ CD4+, la aparición de eventos serios no marcadores de SIDA es relativamente más frecuente en estos pacientes.

De modo que los pacientes sin un marcado deterioro inmunológico (recuentos de linfocitos T CD4+ de más de $200 / \mathrm{mm} 3$ ) presentan un riesgo muy bajo de presentar enfermedades oportunistas pero mantienen una mayor probabilidad de padecer un evento serio no marcador de SIDA que la población general. Este hecho ha llevado a plantear la posibilidad de un inicio anticipado del tratamiento antirretroviral con la intención de disminuir este riesgo. Y si bien aún no existen evidencias claras que sustenten estas recomendaciones, las Guías de tratamiento antirretroviral tanto en nuestro país como en varias partes del mundo reflejan esta posición.

Por otro lado, recientemente se ha demostrado de manera categórica la relación entre el tratamiento y la prevención, en el sentido que el mayor uso de tratamiento antirretroviral en una población se asocia con el descenso del número de nuevos diagnósticos de infección por VIH. Esto agrega una consideración social a la discusión de cuándo se debe comenzar el tratamiento antirretroviral, dado que la menor circulación de virus en una comunidad reduce el riesgo de nuevos contagios. En esta ecuación también influye el hecho que en los últimos años la disponibilidad de nuevas drogas antirretrovirales se ha 
ido incrementando, así como también la disponibilidad de coformulaciones que contribuyen a la simplicidad de los tratamientos y favorecen la adherencia.

La simplificación de los tratamientos en conjunto y su mayor tolerancia constituyen los pilares en que se basa la difusión y el éxito del tratamiento antirretroviral actual, a la vez que contribuyen a la idea de la terapéutica precoz.

En la Argentina hay en la actualidad más de 50.000 personas bajo tratamiento antirretroviral provisto por ley en forma gratuita. Al mismo tiempo se estima que existen alrededor de 110.000 personas infectadas por el VIH, de las cuales alrededor de 40.000 desconoce su condición. Esta realidad reafirma la necesidad de profundizar los trabajos de educación y concientización orientados a promover el diagnóstico precoz y la retención de los pacientes dentro del sistema de salud, y a la vez manteniendo un alto nivel de alerta para interpretar cada consulta de un paciente a cualquier instancia del sistema de salud como una ventana de oportunidad para promover la realización del análisis de VIH voluntario, consentido y con el consejo correspondiente.

\section{Trabajo en equipo}

Desde los inicios de la epidemia se comprendió que la difícil situación determinada por la inmunodeficiencia adquirida requería de un enfoque multidisciplinario para su abordaje.

Con la aparición de las drogas antirretrovirales en 1987, la complejidad creciente de la terapéutica justificó que se jerarquizara el rol del médico especialista como el punto de referencia fundamental -y verdaderamente "médico de cabecera"- de los pacientes con infección por VIH, coordinando la tarea conjunta del equipo de salud. El ritmo de avance del conocimiento en esta enfermedad - prácticamente inigualado en la actualidad -, determina que se haya constituido casi en una subespecialidad.

Actualmente, no existe, en la mayoría de los casos, el condicionante de la inevitabilidad del desenlace y la impotencia frente a la acción como ocurría en el inicio de la epidemia.

La interrelación entre el médico especialista y otros especialistas de aparición eventual en el curso del manejo del paciente con VIH es hoy bastante definida. Es difícil encontrar inconvenientes asistenciales por la acción ocasional del oftalmólogo, el ginecólogo, el psiquiatra, el neumonólogo, el gastroenterólogo o el cirujano. Sin embargo es importante profundizar en el rol del médico de atención primaria en el marco de la asistencia del paciente con VIH para optimizar esta interrelación a favor del paciente.

\section{Unión estratégica}

En muchos casos los pacientes con VIH, más allá de reconocer al especialista como su médico de cabecera, a la vez realizan consultas periódicas con un médico generalista o de atención primaria. Esto puede suceder porque el paciente ya contaba con un médico de cabecera con anterioridad al diagnóstico de infección por VIH y desea mantener ese vínculo, porque el paciente desea o requiere el seguimiento del médico clínico para el manejo de otras patologías concomitantes al VIH, o porque el sistema de salud del paciente determina la existencia de un médico clínico, de familia o generalista, como médico de cabecera u orientador primario del seguimiento.

Por otro lado existe la posibilidad aún más frecuente de la consulta ocasional del paciente con VIH al médico clínico en el contexto de una intercurrencia.

De esta manera se puede comprender la importancia de la definición del rol del médico de atención primaria en el manejo, tanto periódico como eventual, del paciente con infección por VIH. La diversidad de aspectos relacionados con la patología obliga a la definición de tareas que van mucho más allá del establecimiento y el control del tratamiento antirretroviral, transformando, de esta manera, al médico de atención primaria en un verdadero aliado estratégico en beneficio del paciente.

\section{Espacios de interacción}

Además del indudable carácter multidisciplinario de la tarea de prevención, que incluye principalmente la difusión de las medidas de control de la transmisión, el testeo -con consentimientode todas las personas en riesgo y la protección especial de los grupos vulnerables; existen áreas bien definidas en las que el médico de atención primaria tiene una acción primordial en la lucha contra la epidemia, entendiendo esta enumeración como un punto de partida más que como una definición exhaustiva: el diagnóstico inicial de primoinfección o de infección crónica, el manejo inicial de intercurrencias clínicas, la contribución en el mantenimiento de profilaxis primarias y secundarias, las actividades orientadas al manejo del impacto de la enfermedad en el entorno del paciente, y la participación en el diseño y en el desarrollo de estrategias para favorecer la adherencia.

\section{Diagnóstico}

El diagnóstico tardío de la infección por VIH -desafortunadamente tan frecuente en nuestro país así como también en otros países de América Latina- condiciona indudablemente su evolución posterior, a la vez que favorece la propagación de la epidemia.

\section{La primoinfección}

A diferencia de lo que se creía en un principio, la primoinfección por VIH presenta en la mayoría de los casos (entre 66 y 90\%) síntomas que muchas veces motivan una consulta médica. Esta consulta, en la enorme mayoría de los casos, involucra a médicos de atención primaria, clínicos de consultorios externos, sistemas de demanda espontánea o guardias. Se debe considerar este diagnóstico diferencial frente a un cuadro febril de tipo mononucleosiforme, y si bien no existen manifestaciones patognomónicas, un interrogatorio dirigido puede orientar los primeros pasos esenciales para el diagnóstico y evitar la pérdida de una valiosa oportunidad para el control de la enfermedad dado que luego de la infección primaria, la infección puede continuar avanzando en forma silente durante meses o años. En el cuadro 1 se muestran los signos y síntomas más frecuentes de la primoinfección por VIH. La aparición ocasional de infecciones oportunistas tales como candidiasis orofaríngea o eventualmente neumonía por Pneumocystis jiroveci es altamente sugestiva de la etiología retroviral del síndrome. El cuadro clínico deberá ser evaluado en el contexto de otros diagnósticos diferenciales incluyendo infección primaria por virus Epstein Barr o citomegalovirus, rubéola, sarampión, toxoplasmosis aguda, sífilis secundaria, reacción secundaria a drogas o enfermedad gonocócica diseminada.

La consideración de la etiología retroviral del cuadro clínico no implica en ningún caso la inclusión de los análisis específicos para VIH dentro de una "batería" diagnóstica inicial indiscriminada y mucho menos en ausencia de un previo consentimiento expreso por parte del paciente. La posibilidad de resultados falsos positivos en relación a la probabilidad pre-test y la enorme significación emocional que implica el diagnóstico justifican en todos los casos la existencia de un consejo previo y posterior al envío de la muestra para diagnóstico, y la aceptación voluntaria del paciente de la realización del análisis. Sin embargo, el interrogatorio dirigido al paciente, incluyendo antecedentes de análisis de VIH y estado serológico, situaciones de riesgo de transmisión de VIH dentro de los seis meses previos al momento de la consulta y otras manifesta- 
ciones clínicas (diarrea crónica, descenso de peso, otras enfermedades de transmisión sexual) ayudan a establecer una probabilidad diagnóstica.

El diagnóstico de infección primaria por VIH requiere la existencia de replicación viral en ausencia de anticuerpos específicos, por lo cual el test de Elisa será negativo y el Western Blot indeterminado. La confirmación implica la realización de análisis específicos dirigidos a la identificación directa de partículas virales, habitualmente mediante la detección de Antígeno p24 o PCR cuali o cuantitativa.

La sensibilidad de la detección de p24 es de aproximadamente $88 \%$ y la especificidad de $100 \%$, mientras que para los tests de PCR cuantitativa la sensibilidad es cercana a $100 \%$ y la especificidad de $88 \%$. La prueba de PCR suele triplicar en costo a la de Antígeno p24.

La detección de la infección por VIH en su etapa de primoinfección permite establecer el control más preciso del cuadro de inmunodeficiencia, dado que la pérdida de repertorio inmunológico tiene lugar desde el momento mismo del contagio; y posibilita el inicio eventual de un tratamiento antirretroviral en ese momento, valorando cada situación en particular.

Cuadro 1: signos y síntomas habitualmente asociados al síndrome retroviral agudo.

\begin{tabular}{c} 
Frecuencia esperada \\
Fiebre $(96 \%)$ \\
\hline Linfadenopatías $(74 \%)$ \\
\hline Faringitis $(70 \%)$ \\
\hline Rash cutáneo maculopapular (70\%) \\
\hline Mialgias o artralgias (54\%) \\
\hline Diarrea (32\%) \\
\hline Cefalea (32\%) \\
\hline Náuseas y vómitos (27\%) \\
\hline Hepatoesplenomegalia (14\%) \\
\hline Pérdida de peso (13\%) \\
\hline Candidiasis oral (12\%) \\
\hline Manifestaciones neurológicas ${ }^{*}(12 \%)$
\end{tabular}

*Meningoencefalitis o meningitis aséptica, neuropatía periférica o radiculopatía, síndrome de Guillain Barré, parálisis facial, neuritis braquial, alteración cognitiva

\section{La enfermedad crónica asintomática}

Por otro lado, el médico clínico puede tener a su cargo el diagnóstico de la enfermedad asintomática o ser el destinatario de un pedido voluntario de testeo por parte del paciente. También en estos casos el interrogatorio dirigido y el consejo previo y posterior al resultado del análisis son elementos fundamentales para un diagnóstico adecuado.

El diagnóstico de infección por VIH es en general el más grave que el paciente ha sufrido y lleva implícita una gran carga de angustia y preocupación que el médico nunca debe soslayar. En todos los casos se deberá contar con el análisis confirmatorio (ej. Western Blot) antes de certificar al paciente su condición.

La derivación del paciente al médico especialista podrá ser realizada de inmediato o bien podrá esperarse hasta contar con los análisis de carga viral plasmática y recuento de linfocitos T CD4+ que permitan establecer un cuadro de la situación actual del paciente respecto de su enfermedad. En cualquier caso es indudable la importancia de esta primera orientación del paciente en su condición de seropositivo. En el cuadro 2 se incluyen algunas recomendaciones generales para la visita en la que se confirma el diagnóstico de infección por $\mathrm{VIH}$, previa a la primera consulta con el especialista.

Existen dos consideraciones adicionales en relación al diagnóstico de la infección por VIH y el rol del médico de atención primaria. En primer lugar la situación particular del embarazo, que constituye una indicación indiscutida de inicio del tratamiento antirretroviral en pacientes con infección por $\mathrm{VIH}$ dado que la transmisión vertical puede ser disminuida hoy efectivamente a valores menores al $1 \%$. Esto subraya la imperiosa necesidad de fomentar el diagnóstico de la mujer embarazada (en presencia de consentimiento y consejo previo y posterior) que permita tomar a tiempo las medidas adecuadas para reducir al máximo posible el número de niños infectados por $\mathrm{VIH}$. Por otra parte debe desalentarse categóricamente la determinación de serología para VIH en el examen prequirúrgico, dado que a su comprobada inutilidad (muchas veces contribuye incluso a flexibilización de medidas de prevención necesarias) se suma en la mayoría de los casos el cuestionamiento ético derivado de la ausencia del consentimiento y consejo adecuados ya referidos anteriormente.

Cuadro 2: recomendaciones generales para la consulta inicial en que se confirma el diagnóstico.

1) Mostrar al paciente los análisis de la confirmación del diagnóstico. Mantener una actitud respetuosa y cordial durante la entrevista.

2) No sobrecargar al paciente con datos o información que será difícil de procesar en el contexto de la carga emocional de la consulta. Responder

las inquietudes que manifiesta. Afirmar que habrá muchas instancias adicionales en las que podrá encontrar respuesta a sus dudas.

3) Indicar que no es posible establecer un pronóstico hasta que no se cuente con los resultados de los análisis de carga viral y recuento de CD4+ correspondientes.

4) Recomendar que el paciente no establezca conductas de riesgo (relaciones sexuales sin protección de barrera, consumo de drogas intravenosas).

5) Sugerir que posiblemente será mejor contar con mayores datos y haber tenido la entrevista con el especialista antes de comunicar el diagnóstico a familiares y amigos (evaluar cada caso individual).

6) Reafirmar que la convivencia y el contacto cotidiano no presentan riesgo de transmisión del VIH, cuidando el contacto con sangre y fluidos sexuales.

7) Recomendar que el paciente no intente precipitar acciones y decisiones. Plantear metas progresivas posibles de cumplir. En principio, el cuidado personal, la consulta con el especialista y los análisis correspondientes.

8) No hay restricciones dietéticas específicas en este momento. Si el paciente solicita actividades para realizar en este período, la gimnasia aeróbica dos o tres veces por semana y la reducción o supresión del tabaquismo, son medidas con impacto potencial sobre la inmunidad.

9) Ofrecer al paciente formas de contacto telefónico o personal para consultas.

10) Confirmar reiteradamente en el curso de la visita que, independientemente del estado inmunológico del paciente, siempre hay mucho por hacer y que la medicación disponible puede modificar sustancialmente el pronóstico aun en casos de infección por VIH avanzada 


\section{Aspectos de manejo clínico}

En distintas ocasiones el paciente con $\mathrm{VIH}$ puede concurrir a una consulta en guardia externa o consultorio de demanda espontánea ya sea por intercurrencias relacionadas con el VIH o su tratamiento, o bien por eventos clínicos no necesariamente relacionados con su enfermedad. El manejo inicial del paciente en estas situaciones muchas veces depende del médico de atención primaria o de guardia

Se debe recordar que el conocimiento del nivel de células CD4+ es fundamental para afirmar o relegar la sospecha clínica de un evento de progresión relacionado con la infección por $\mathrm{VIH}$. En el cuadro 3 se muestra un resumen del riesgo de aparición de infecciones oportunistas en relación con el estadío de la enfermedad por VIH.

Cuadro 3: riesgo de progresión de enfermedad por el virus de la inmunodeficiencia adquirida.

\begin{tabular}{|c|c|c|c|}
\hline Carga viral basal (Copias/mL) & $\begin{array}{l}\text { Recuento basal } \\
\left.\text { de CD4+( } / \mathrm{mm}^{3}\right)\end{array}$ & $\begin{array}{c}\text { Riesgo de SIDA (\%) } \\
\text { A los } 3 \text { años }\end{array}$ & $\begin{array}{c}\text { Riesgo de SIDA (\%) } \\
\text { A los } 9 \text { años }\end{array}$ \\
\hline \multirow[t]{2}{*}{ Menos de 500} & Menos de 750 & 3,7 & 22,3 \\
\hline & Más de 750 & 0,0 & 3,6 \\
\hline \multirow[t]{3}{*}{10001 a 30000} & Menos de 350 & 40,1 & 86,2 \\
\hline & 351 a 750 & 16,1 & 76,3 \\
\hline & Más de 750 & 9,5 & 62,4 \\
\hline \multirow[t]{4}{*}{ Más de 30000} & Menos de 200 & 85,5 & 100,0 \\
\hline & 201 a 350 & 64,4 & 92,9 \\
\hline & 351 a 500 & 47,9 & 94,4 \\
\hline & Más de 500 & 32,6 & 76,3 \\
\hline
\end{tabular}

SIDA definido por la aparición de Infecciones Oportunistas, sin incluir descenso de CD4+ por debajo de 200/mm³.

Es importante que el médico clínico se encuentre advertido de cuáles son las principales manifestaciones clínicas de toxicidad de los distintos fármacos antirretrovirales, dado que pueden constituirse en motivo de consulta de urgencia. En el cuadro 4 se muestran las principales drogas antirretrovirales utilizadas en la actualidad.

Cuadro 4: drogas antirretrovirales y dosis habituales para adultos.

\begin{tabular}{|c|c|c|}
\hline FAMILIA & DROGA & DOSIS \\
\hline $\begin{array}{l}\text { Inhibidores nucleósidos de } \\
\text { transcriptasa reversa } \\
\text { (ITRN) }\end{array}$ & $\begin{array}{l}\text { Abacavir (ABC) } \\
\text { Didanosina (ddl) } \\
\text { Lamivudina (3TC) } \\
\text { Emtricitabina (FTC) } \\
\text { Stavudina (d4T) } \\
\text { Zidovudina (AZT) }\end{array}$ & $\begin{array}{c}300 \mathrm{mg} \mathrm{c} / 12 \mathrm{~h} \\
(>60 \mathrm{~kg}): 200 \mathrm{mg} \mathrm{c} / 12 \mathrm{~h} \text { ó } 400 \mathrm{mg} / \mathrm{día} \\
(<60 \mathrm{~kg}): 125 \mathrm{mg} \mathrm{c} / 12 \mathrm{~h} \text { ó } 250 \mathrm{mg} / \mathrm{día} \\
150 \mathrm{mg} \mathrm{c} / 12 \mathrm{~h} \\
200 \mathrm{mg} / \mathrm{día} \\
(>60 \mathrm{~kg}): 40 \mathrm{mg} \mathrm{c} / 12 \mathrm{~h} \\
(<60 \mathrm{~kg}): 30 \mathrm{mg} \mathrm{c} / 12 \mathrm{~h} \\
500 \text { a } 600 \mathrm{mg} / \mathrm{dia}\end{array}$ \\
\hline $\begin{array}{l}\text { Inhibidores nucleótidos de } \\
\text { transcriptasa reversa }\end{array}$ & Tenofovir (TFV) & $300 \mathrm{mg} / \mathrm{di} a$ \\
\hline $\begin{array}{l}\text { Inhibidores no nucleósidos de } \\
\text { transcriptasa reversa } \\
\text { (ITRNN) }\end{array}$ & $\begin{array}{c}\text { Efavirenz (EFV) } \\
\text { Nevirapina (NVP) } \\
\text { Etravirina (ETV) } \\
\end{array}$ & $\begin{array}{c}600 \mathrm{mg} / \text { día } \\
200 \mathrm{mg} / \text { día, luego } 200 \mathrm{mg} \mathrm{c} / 12 \mathrm{~h} \\
200 \mathrm{mg} \mathrm{c} / 12 \mathrm{~h}\end{array}$ \\
\hline $\begin{array}{l}\text { Inhibidores de proteasa } \\
\text { (IP) }\end{array}$ & $\begin{array}{c}\text { Potenciación con Ritonavir } \\
\text { Fosamprenavir (APV) } \\
\text { Atazanavir } \\
\text { Lopinavir/r (LPV) } \\
\text { Saquinavir }\end{array}$ & $\begin{array}{l}\text { Habitualmente } 100 \text { o } 200 \mathrm{mg} / \text { día } \\
700 \mathrm{mg} \mathrm{c} / 12 \mathrm{~h} \\
300 \text { o } 400 \mathrm{mg} / \mathrm{día} \\
200 \mathrm{mg} \mathrm{c} / 12 / \mathrm{h} \\
500 \mathrm{mg} \mathrm{c} / 12 \mathrm{~h}\end{array}$ \\
\hline Inhibidores de Intetgrasa & Raltegravir & $400 \mathrm{mg} \mathrm{c} / 12 \mathrm{hs}$ \\
\hline Inhibidores de CCR-5 & Maraviroc & 150 o $300 \mathrm{mg} \mathrm{c} / 12 \mathrm{~h}$ \\
\hline
\end{tabular}

Inhibidores nucleósidos de la transcriptasa reversa (ITRN) Hasta un $10 \%$ de los pacientes pueden presentar náuseas, vómitos, astenia y cefalea en los primeros siete a diez días luego del inicio de un régimen que contiene zidovudina (AZT). Habitualmente el especialista advierte al paciente sobre esta posibilidad, resaltando que por lo general, estas manifestaciones no son severas y son autolimitadas. Casi todos los pacientes que reciben zidovudina presentarán macrocitosis y eventualmente una reducción del número de glóbulos rojos sin que deba tomarse una conducta al respecto en la mayoría de los casos. Incluso se ha sugerido la eventual utilidad de la macrocitosis como prueba de la adherencia a la toma de la medicación. El uso de didanosina (ddl) puede propiciar la aparición de pancreatitis, particularmente en pacientes con antecedentes de consumo de alcohol o enfermedad biliar. Alrededor de $4 \%$ de los pacientes que reciben abacavir (ABC) pueden presentar una reacción de hipersensibilidad caracterizada por fiebre, erupción cutánea, cefalea y síntomas gastrointestinales, habitualmente dentro de las dos primeras semanas de tratamiento. Este síndrome de hipersensibilidad requiere la 
suspensión del abacavir y contraindica su re exposición posterior. En la actualidad se dispone de un análisis farmacogenético (detección de HLAB*5701) que permite predecir la aparición del síndrome de hipersensibilidad por abacavir por lo que se recomienda su realización con anterioridad a la indicación de este fármaco. La lamivudina y la emtricitabina son drogas muy similares entre sí que forman parte de la mayoría de los regímenes antirretrovirales de combinación y presentan perfiles de tolerancia y toxicidad muy favorables.

El tenofovir disoproxil fumarato es un fármaco análogo de nucleótido que se utiliza hoy como parte de las primeras líneas de tratamiento antirretroviral en la mayoría de los países del mundo. Es un fármaco muy bien tolerado y con escasos efectos adversos agudos. Sin embargo, a mediano y largo plazo, puede condicionar la aparición de osteopenia-osteoporosis, y trastornos de la función renal -alteraciones de la función tubular proximal o deterioro del filtrado glomerular- en particular en pacientes con antecedentes o con otros factores de riesgo de insuficiencia renal crónica.

La administración de didanosina (ddl), stavudina (d4T) o zalcitabina (ddC) -hoy no recomendada- puede inducir la aparición de neuropatía periférica en 10 a $20 \%$ de los pacientes. Esta neuropatía es sensitiva, bilateral, habitualmente muy dolorosa y de desarrollo relativamente lento. El diagnóstico clínico de neuropatía justifica la solicitud de un electromiograma, y más allá del manejo inicial eventual con analgésicos, requerirá de la intervención del especialista para las decisiones referidas a la modificación del tratamiento antirretroviral.

La acidosis láctica es una intercurrencia poco frecuente pero muy trascendente del uso de cualquier droga de la familia de los nucleósidos, dadas sus consecuencias potencialmente fatales. Considerando que prácticamente todos los regímenes antirretrovirales actualmente vigentes incluyen al menos una droga de esta familia, virtualmente cualquier paciente bajo tratamiento se encuentra en riesgo, aunque este es mayor con el uso de análogos de timidina (zidovudina, stavudina) y mínimo para otros fármacos como tenofovir, abacavir y lamivudina, Frente a un cuadro de dolor abdominal agudo, náuseas y vómitos en el que no se halla la causa con los exámenes iniciales, siempre debe considerarse la acidosis láctica dentro de los diagnósticos diferenciales, en especial si se detecta taquipnea en el examen físico. La elevada mortalidad asociada con este síndrome en ausencia de tratamiento (70 a $80 \%$ ) subraya la importancia del manejo inicial del paciente, en particular mediante la monitorización permanente y la reposición de líquidos y bicarbonato. El régimen antirretroviral debe interrumpirse de inmediato frente a la aparición de este cuadro.

\section{Inhibidores de transcriptasa inversa no nucleósidos (ITRNN)}

La nevirapina y el efavirenz son los inhibidores no nucleósidos más utilizados en la actualidad, a los que más recientemente se agregó la etravirina. En general son inductores de CYP3A4. La toxicidad principal común de esta familia de drogas es la hipersensibilidad, manifestada como erupción cutánea. Esta erupción aparece habitualmente dentro de las dos o tres semanas desde el inicio del tratamiento. Es importante considerar que solo requieren la suspensión del fármaco aquellos pacientes en que se observan síntomas significativos (fiebre mayor a $39^{\circ} \mathrm{C}$, astenia, prurito de difícil manejo), compromiso hepático o de mucosas. El manejo inicial puede realizarse con antihistamínicos (ver restricciones en interacciones) o con tratamientos locales. En algunas ocasiones el síndrome de hipersensibilidad se acompaña de la elevación de los niveles de transaminasas, cuya magnitud también influirá en la decisión de la suspensión del tratamiento.

El efavirenz presenta una toxicidad distintiva sobre el sistema nervioso central, que incluye la aparición de mareos, insomnio, dificultad para concentrarse, irritabilidad y sueños vívidos o pesadillas. El paciente debe ser advertido de la posibilidad de aparición de estos efectos, y se recomienda que la toma de la dosis sea realizada antes de acostarse. Habitualmente, estos síntomas son leves y transitorios y mejoran en el curso de los primeros dos meses de tratamiento, aun sin tratamiento específico. Solo los cuadros más severos justifican la interrupción del fármaco. La nevirapina puede producir toxicidad hepática manifiesta por elevación de las transaminasas, particularmente en pacientes que inician tratamiento con recuentos relativamente elevados de linfocitos T-CD4+ (más de 250/mm3 en mujeres o más de $400 / \mathrm{mm} 3$ en varones).

\section{Inhibidores de proteasa (IP)}

Son drogas de rango terapéutico estrecho y habitualmente bloqueantes muy potentes de CYP3A4 que componen, junto con los inhibidores nucleósidos, la versión original de los tratamientos de alta eficacia. Existen manifestaciones de toxicidad comunes a todas las drogas de la familia, en particular la intolerancia gastrointestinal y la diarrea, y en menor medida las cefaleas y la elevación de transaminasas.

El otro efecto secundario característico de clase de los inhibidores de proteasa -utilizados en conjunto con inhibidores nucleósidos- es el síndrome de lipodistrofia, que incluye cambios en la distribución de grasa del organismo, hipercolesterolemia, hipertrigliceridemia y resistencia periférica a la insulina o más raramente diabetes.

La diarrea es un efecto adverso frecuentemente asociado con el uso de saquinavir y ritonavir, y en ocasiones puede justificar la consulta en guardia, sobre todo durante las primeras semanas del tratamiento. El manejo agudo del episodio puede realizarse con loperamida o crema de bismuto, o bien mediante el agregado de fibras a la dieta, y normalmente no requiere de la suspensión de la droga.

En la actualidad se emplea el ritonavir en bajas dosis (100 a $200 \mathrm{mg} \mathrm{c} / 12 \mathrm{~h}$ ) en combinación con otros inhibidores de proteasa con el objetivo de aumentar su concentración plasmática por bloqueo metabólico (potenciación o "boosting"). En estos casos la toxicidad dosis-dependiente del inhibidor de proteasa que se combina con ritonavir verá aumentada su posibilidad de aparición, por lo cual se utilizan menores dosis en la combinación. El lopinavir es uno de los inhibidores de proteasa disponibles en la actualidad. Se utiliza en conjunto con bajas dosis de ritonavir y los principales efectos secundarios que produce incluyen diarrea, intolerancia digestiva e hiperlipidemias. El atazanavir es uno de los inhibidores de proteasa más utilizados. Tiene una dosificación conveniente -una sola toma diariay es un fármaco bien tolerado. No determina la aparición de hiperlipidemias aunque puede asociarse con un leve riesgo de intolerancia a la glucosa. El principal efecto secundario es la hiperbilirrubinemia indirecta, producida por la inhibición de la enzima UGT1A1, que establece un inconveniente más de carácter estético que funcional.

\section{Otros fármacos}

Dentro de las drogas de aparición más reciente se destacan dos fármacos representantes de nuevas familias.

El raltegravir es el primer inhibidor de integrasa disponible comercialmente. Su toxicidad a corto y mediano plazo parece ser muy leve, con cefaleas y náuseas transitorias.

El maraviroc es un inhibidor del correceptor CCR-5, vital para el ingreso del virus a la célula. Es también un fármaco muy bien tolerado que raramente puede ocasionar erupción cutánea y vértigo. En el cuadro 5 se muestran motivos de consulta de urgencia vinculados con el tratamiento antirretroviral así como sugerencias para su manejo inicial. 
Cuadro 5: motivos de consulta más frecuentes secundarios a tratamiento antirretroviral, y sugerencias para el manejo inicial.

\begin{tabular}{|c|c|c|}
\hline Manifestación & Drogas habitualmente asociadas & Manejo inicial \\
\hline Erupción cutánea & $\begin{array}{c}\text { Nevirapina } \\
\text { Efavirenz } \\
\text { Abacavir } \\
\text { Fosamprenavir } \\
\text { Maraviroc } \\
\end{array}$ & $\begin{array}{c}\text { Abacavir: suspensión } \\
\text { Resto: Antihistamínicos (no terfenadina ni astemizol), } \\
\text { control clínico. Suspensión en caso de compromiso } \\
\text { mucoso o síntomas severos }\end{array}$ \\
\hline Cefaleas & $\begin{array}{c}\text { Zidovudina } \\
\text { Inhibidores de Proteasa } \\
\text { Raltegravir }\end{array}$ & Manejo sintomático. Habitualmente autolimitadas \\
\hline Náuseas y vómitos & $\begin{array}{c}\text { Zidovudina } \\
\text { Inhibidores de Proteasa } \\
\text { Raltegravir }\end{array}$ & Manejo sintomático con metoclopramida si es necesario \\
\hline Diarrea & $\begin{array}{l}\text { Ritonavir } \\
\text { Nelfinavir }\end{array}$ & $\begin{array}{l}\text { Dieta astringente, eventualmente loperamida o crema } \\
\text { de bismuto }\end{array}$ \\
\hline Dolor abdominal & Inhibidores Nucleósidos & $\begin{array}{c}\text { Descartar inicialmente patología intraabdominal de } \\
\text { urgencia (perforación, pancreatitis). Considerar la } \\
\text { posibilidad de acidosis láctica (dosaje de ácido láctico y } \\
\text { bicarbonato) }\end{array}$ \\
\hline \multicolumn{2}{|c|}{$\begin{array}{l}\text { Criterio para la suspensión del tratamiento } \\
\text { En aquellos casos en que, de acuerdo con la gravedad del } \\
\text { cuadro de toxicidad referido anteriormente, se decida la sus- } \\
\text { pensión inmediata de alguno de los fármacos antirretrovirales, } \\
\text { deberá suspenderse todo el régimen de combinación que } \\
\text { recibe el paciente, para evitar la exposición de la carga viral } \\
\text { remanente a concentraciones subóptimas de fármacos, lo que } \\
\text { puede determinar el desarrollo de resistencia virológica. }\end{array}$} & $\begin{array}{l}\text { guardias o consultorios de demanda espontánea por intercurren- } \\
\text { cias clínicas no son medicados o reciben dosis subterapéuticas de } \\
\text { fármacos, por el temor de las interacciones. En este sentido es } \\
\text { importante reconocer que: a) la inducción farmacocinética (como } \\
\text { la observada por el uso de drogas como nevirapina o efavirenz) es } \\
\text { un fenómeno de desarrollo lento, ya que demanda la síntesis de } \\
\text { proteínas y habitualmente no complica la te-rapéutica eventual de } \\
\text { una intercurrencia aguda; b) el bloqueo del metabolismo por inhibi- } \\
\text { ción de CYP (tal como el observado con el uso de inhibidores de } \\
\text { proteasa) es un fenómeno más rápido pero cuyas consecuencias } \\
\text { en general se observan con la administración sostenida de fárma- } \\
\text { cos que sean sustratos de las isoenzimas correspondientes; c) si } \\
\text { bien la lista de drogas con interacción potencial es extensa, la } \\
\text { mayoría de ellas requiere control clínico adicional, monitoreo o } \\
\text { modificación eventual de dosis; y solo un número reducido de ellas } \\
\text { se encuentra formalmente contraindicada para el uso en pacientes } \\
\text { bajo tratamiento antirretroviral de alta eficacia. Estas serían en } \\
\text { realidad las drogas que el médico que asiste a un paciente con } \\
\text { infección por VIH por una intercurrencia debería cuidarse de no } \\
\text { indicar. En el cuadro } 6 \text { se enumeran las drogas con contraindi- } \\
\text { cación absoluta de ser administradas en pacientes bajo tratamien- } \\
\text { to antirretroviral. Para el listado exhaustivo de las distintas drogas } \\
\text { y sus interacciones potenciales se remite al lector a otras referen- } \\
\text { cias. }\end{array}$ \\
\hline
\end{tabular}

Cuadro 6: drogas contraindicadas en pacientes bajo tratamiento antirretroviral de alta eficacia (en orden alfabético.

\begin{tabular}{l|l}
\multicolumn{1}{c|}{ PACIENTES CON ITRNN } & \multicolumn{1}{c}{ PACIENTES CON IP } \\
\hline Astemizol, terfenadina & Benzodiazepinas (excepto lorazepam) \\
cisapride, & amiodarona, bepridil, flecainida propafenona, quinidina \\
ergotamina y derivados del ergot, & astemizol, terfenadina \\
ketoconazol, & cisapride, \\
midazolam, triazolam & ergotamina y derivados del ergot, \\
rifampicina & lovastatina, simvastatina \\
& pimozida, \\
& rifampicina, \\
\hline
\end{tabular}

\section{ITRNN: Inhibidores de transcriptasa inversa no-nucleósidos, IP: Inhibidores de proteasa.}

\section{Profilaxis primarias y secundarias}

La infección por VIH sin tratamiento adecuado conduce a la inmunodeficiencia que caracteriza al cuadro avanzado, con mayor riesgo de aparición o reactivación de infecciones opor- tunistas. A pesar de los avances obtenidos con el tratamiento antirretroviral de alta eficacia en términos de reducción de la morbilidad y mortalidad, el diagnóstico tardío que aun caracteriza a los países en desarrollo exige el mantenimiento de la 
atención en el diagnóstico preciso y la prevención de las infecciones oportunistas. En general los riesgos aparecen con recuentos de células CD4+ por debajo de $200 / \mathrm{mm}^{3}$, siendo este valor el punto de corte para el inicio de profilaxis para neumonía por P. jiroveci. Recuentos menores justifican el inicio de la profilaxis para to-xoplasmosis y Mycobacterium avium. La reconstitución inmunológica observada con el uso del tratamiento antirretroviral permite la suspensión de las profilaxis prácticamente $\sin$ riesgo de recaídas. En el cuadro 7 se especifican los criterios actuales de profilaxis de las infecciones oportunistas más frecuentes y en el cuadro 8 se incluyen los regímenes recomendados.

La consideración de estos criterios con el fin de minimizar el riesgo de los pacientes sin exponerlos al uso de medicación innecesaria, así como el control del cumplimiento de las profilaxis en aquellos que tienen indicación precisa son, sin dudas, áreas de responsabilidad primordial del médico generalista 0 de atención primaria.

Cuadro 7: inicio y suspensión de profilaxis para infecciones oportunistas en pacientes con infección por VIH.

\begin{tabular}{|c|c|c|c|c|}
\hline Infección oportunista & $\begin{array}{l}\text { Inicio de profilaxis } \\
\text { primaria }\end{array}$ & $\begin{array}{l}\text { Suspensión de } \\
\text { profilaxis primaria }\end{array}$ & $\begin{array}{l}\text { Inicio de profilaxis } \\
\text { secundaria }\end{array}$ & $\begin{array}{c}\text { Suspensión de } \\
\text { profilaxis secundaria }\end{array}$ \\
\hline $\begin{array}{l}\text { Neumonía por } \\
\text { Pneumocystis jiroveci }\end{array}$ & $\begin{array}{l}\text { Recuento de CD4+ } \\
\text { menor a } 200 / \mathrm{mm}^{3} \text { ó } \\
\text { candidiasis }\end{array}$ & $\begin{array}{l}\text { Recuento de CD4+ } \\
\text { mayor a } 200 / \mathrm{mm}^{3} \text { por } \\
\text { más de tres meses }\end{array}$ & $\begin{array}{l}\text { PCP previa. Descenso de } \\
\text { CD4+ a menos de } 200 / \mathrm{mm}^{3} \\
\text { Toxo SNC ó descensos de }\end{array}$ & $\begin{array}{c}\text { Recuento de CD } 4+\text { mayor a } 200 / \mathrm{mm}^{3} \text { por } \\
\text { más de tres meses }\end{array}$ \\
\hline Toxoplasmosis & $\begin{array}{c}\text { Recuento de CD4+ } \\
\text { menor a } 100 / \mathrm{mm}^{3} \text { y } \\
\text { anticuerpos anti } \\
\text { toxoplasma positivos }\end{array}$ & $\begin{array}{l}\text { Recuento de CD4+ } \\
\text { mayor a } 200 / \mathrm{mm}^{3} \text { por } \\
\text { más de tres meses }\end{array}$ & $\begin{array}{l}\text { CD4+ a menos de } 200 / \mathrm{mm}^{3} \\
\text { MAC documentada. }\end{array}$ & $\begin{array}{l}\text { Recuento de CD4+ mayor de } 200 / \mathrm{mm}^{3} \text { por } \\
\text { más de seis meses en paciente asintomático } \\
\text { y con tratamiento completo inicial }\end{array}$ \\
\hline $\begin{array}{l}\text { Mycobacterium } \\
\text { avium-intracelulare }\end{array}$ & $\begin{array}{l}\text { Recuento de CD4+ } \\
\text { menor a } 50 / \mathrm{mm}^{3}\end{array}$ & $\begin{array}{l}\text { Recuento de CD4+ } \\
\text { mayor a } 100 / \mathrm{mm}^{3} \text { por } \\
\text { más de tres meses }\end{array}$ & $\begin{array}{l}\text { Descensos de CD4+ a } \\
\text { menos de } 100 / \mathrm{mm}^{3}\end{array}$ & $\begin{array}{l}\text { Recuento de CD4+ mayor de } 100 / \mathrm{mm}^{3} \text { por } \\
\text { más de seis meses, en paciente asintomático } \\
\text { con tratamiento completo inicial ( } 12 \text { meses). }\end{array}$ \\
\hline Criptococosis & No & NA & $\begin{array}{l}\text { Enfermedad documentada. } \\
\text { Descensos CD4+ a } \\
<100-200 / \mathrm{mm}^{3}\end{array}$ & $\begin{array}{l}\mathrm{CD} 4+>100-200 / \mathrm{mm}^{3} \text { por } 6 \text { meses, } \\
\text { asintomático, tratamiento completo inicial }\end{array}$ \\
\hline Histoplasmosis-coccidiomicosis & No & NA & Enfermedad documentada & No hay criterios para suspensión \\
\hline Citomegalovirus & No & NA & Enfermedad documentada & $\begin{array}{l}\text { Recuento de CD4+ mayor a } 100 \text { ó } 150 / \mathrm{mm}^{3} \\
\text { por más de seis meses, en paciente } \\
\text { asintomático, sin lesiones activas en fondo } \\
\text { del ojo, sin lesión bilateral y sin lesión } \\
\text { perimacular. }\end{array}$ \\
\hline
\end{tabular}

PCP: neumonía por Pneumocystis jiroveci, Toxo SNC: toxoplasmosis del Sistema Nervioso Central, MAC: Infección por Mycobacterium aviumintracelulare; NA: no aplicable.

Cuadro 8: regímenes profilácticos recomendados.

\begin{tabular}{|c|c|c|}
\hline Patógeno & Elección & Alternativa \\
\hline $\begin{array}{l}\text { P. jiroveci } \\
\text { (antes P. carinii) }\end{array}$ & $\begin{array}{l}\text { TMS: 160mg TMP/ } \\
\text { 800mg SMX/día }\end{array}$ & $\begin{array}{l}\text { Dapsona } 100 \mathrm{mg} / \mathrm{día} \text { ó } \\
\text { Dapsona } 50 \mathrm{mg} / \mathrm{día}+\text { Pirimetamina } 50 \mathrm{mg} / \mathrm{sem} \text { + leucovorina } 25 \mathrm{mg} / \mathrm{sem} \text { ó } \\
\text { Dapsona } 200 \mathrm{mg}+\text { leucovorina } 25 \mathrm{mg} / \mathrm{sem} \\
\text { TMS: } 160 / 800 \mathrm{mg} 3 \text { veces por sem. }\end{array}$ \\
\hline Toxoplasmosis & $\begin{array}{l}\text { TMS: 160mg TMP/ } \\
\text { 800mg SMX/día }\end{array}$ & Dapsona 50 mg/día + Pirimetamina 50 mg/sem + leucovorina 25 mg/sem \\
\hline $\begin{array}{l}\text { Toxoplasmosis } \\
\text { (profilaxis secundaria) }\end{array}$ & $\begin{array}{l}\text { Sulfadiazina } 500 \text { a } 1000 \mathrm{mg} \text { c/6h + } \\
\text { Pirimetamina } 25 \text { a } 50 \mathrm{mg} / \mathrm{día}+ \\
\text { leucovorina } 10 \text { a } 25 \mathrm{mg} / \mathrm{día}\end{array}$ & $\begin{array}{l}\text { Clindamicina } 300 \text { a } 450 \mathrm{mg} \text { c/6 a } 8 \mathrm{~h}+\text { Pirimetamina } 25 \text { a } 50 \mathrm{mg} / \mathrm{día}+ \\
\text { leucovorina } 10 \text { a } 25 \mathrm{mg} / \mathrm{dí} \text { + }\end{array}$ \\
\hline Mycobacterium avium complex & $\begin{array}{l}\text { Azitromicina } 1200 \mathrm{mg} / \mathrm{sem} \text { ó } \\
\text { Claritromicina } 500 \mathrm{mg} \mathrm{c} / 12 \mathrm{~h}\end{array}$ & Rifabutina $300 \mathrm{mg} / \mathrm{día}$ \\
\hline $\begin{array}{l}\text { Mycobacterium avium complex } \\
\text { (profilaxis secundaria) }\end{array}$ & $\begin{array}{l}\text { Claritromicina } 500 \mathrm{mg} \mathrm{c} / 12 \mathrm{~h}+ \\
\text { etambutol } 15 \mathrm{mg} / \mathrm{kg} \otimes \text { rifabutina } \\
300 \mathrm{mg} / \mathrm{di} a\end{array}$ & Azitromicina $500 \mathrm{mg} / \mathrm{día}+$ etambutol $15 \mathrm{mg} / \mathrm{kg} \otimes$ rifabutina $300 \mathrm{mg} / \mathrm{día}$ \\
\hline $\begin{array}{l}\text { Mycobacterium tuberculosis } \\
\text { (PPD +) }\end{array}$ & $\begin{array}{l}\text { Isoniazida } 300 \mathrm{mg} / \mathrm{día}+\text { piridoxina } \\
50 \mathrm{mg} / \mathrm{dia} \text { x seis a nueve meses } \\
\text { Isoniazida } 900 \mathrm{mg}+\text { piridoxina } 100 \mathrm{mg} \\
\text { dos veces/sem } \times \text { nueve meses }\end{array}$ & Rifampicina $600 \mathrm{mg}+$ pirazinamida 15 a $20 \mathrm{mg} / \mathrm{kg} / \mathrm{dia}$ por dos meses \\
\hline $\begin{array}{l}\text { Criptococosis (profilaxis } \\
\text { secundaria) }\end{array}$ & Fluconazol 200mg/día & Anfotericina B intravenosa 0,6 a 1mg/kg/semana ó Itraconazol 200mg/día \\
\hline $\begin{array}{l}\text { CMV } \\
\text { (profilaxis secundaria) }\end{array}$ & Valganciclovir $900 \mathrm{mg} / \mathrm{dí}$ & Ganciclovir 5 a $6 \mathrm{mg} / \mathrm{kg} / \mathrm{d}$ ía cinco veces $/ \mathrm{sem}$. \\
\hline
\end{tabular}

TMS: trimetoprima-sulfametoxazol, TMP: trimetoprima, SMX: sulfametoxazol, CMV: citomegalovirus (retinitis) 


\section{Vacunas}

Todos los pacientes adultos con infección por VIH deben recibir cada diez años el refuerzo correspondiente de la vacuna antitetánica (o doble de adultos). Asimismo, deben recibir la vacuna recombinante para hepatitis $B$ y para hepatitis $A$ aquellos pacientes con serología negativa para dichas infecciones. También se recomienda la vacunación para enfermedad neumocóccica aunque algunas veces la oportunidad de la vacunación se difiere hasta obtener algún resultado inmunológico del tratamiento antirretroviral en pacientes con infección avanzada. Asimismo está recomendada la vacunación anual para influenza.

\section{Impacto personal y contexto}

El diagnóstico de infección por VIH implica habitualmente un riesgo de estigmatización para el paciente, el cual, sumado a la indudable gravedad de la enfermedad, le impone severas exigencias tanto para asumir personalmente su nueva condición, como para establecer alianzas en su medio social y afectivo. Aconsejar es fundamental en ese momento, y es probable que el paciente desee una opinión adicional a la del especialista a quien ha conocido recientemente. Prestar atención al impacto emocional siempre es de gran importancia, pero es vital durante las primeras semanas o meses luego del diagnóstico, hasta que se consiguen establecer prioridades individuales, metas terapéuticas y planes estratégicos para enfrentar la enfermedad.

La revisión de los modos de transmisión, que permite al pacientes proteger a sus convivientes y contactos, el mejor momento para informar el diagnóstico a los familiares y amigos, y la sucesión de metas alcanzables que contribuyan a modificar su pronóstico son áreas cruciales para dialogar y desarrollar individualmente con el paciente.

\section{Estrategias para fomentar la adherencia}

Frente a un escenario de disponibilidad gratuita de medicación como en nuestro medio, la adherencia al tratamiento antirretroviral es seguramente el aspecto más crítico que condiciona el éxito a largo plazo en el manejo de la infección por VIH.

Se sabe que la adherencia a los tratamientos crónicos tiende a decaer con el tiempo, y para el caso de las drogas antirretrovirales se ha informado que la necesidad de adherencia para obtener la máxima eficacia de un régimen debería ser superior al $95 \%$. Una de las principales herramientas para favorecer la adherencia es la disponibilidad de tratamientos más simples. Respecto de este punto se han observado recientemente avances significativos con la disponibilidad de drogas administradas una vez por día (didanosina, emtricitabina, tenofovir, efavirenz, atazanavir, fos-amprenavir), de combinaciones de drogas en la misma formulación (zidovudina-lamivudina-abacavir, lopinavir-ritonavir, emtricitabina-tenofovir, emtricitabinatenofovir-efavirenz) y a través de los regímenes de inhibidores de proteasa potenciados por bajas dosis de ritonavir ("boosting"). Esta situación podría contribuir a disminuir en cierta medida una exigencia de adherencia de improbable cumplimiento en el largo plazo mediante el uso de regímenes algo más "permisivos".

Aun así la trascendencia del cumplimiento del régimen terapéutico justifica que cada visita del paciente al especialista, al médico de atención primaria o a la farmacia, deba ser interpretada como una oportunidad para el control y el refuerzo de la adherencia. Este control no debe tener un carácter punitivo sino que debe orientarse a la búsqueda de herramientas individuales que permitan superar los problemas específicos de la toma de la medicación.

La depresión, el consumo activo de alcohol o de drogas intravenosas y la falta de comprensión por bajo nivel de escolaridad se encuentran dentro del número limitado de factores asociados en forma consistente a la disminución de la adherencia a regímenes antirretrovirales.

Existen diferentes métodos de evaluar la adherencia, aunque los más sencillos de aplicar en nuestro medio son los interrogatorios dirigidos, los cuestionarios de autoevaluación, el recuento periódico de comprimidos y los registros de provisión de la farmacia. En el cuadro 9 se sugieren medidas con potencial impacto sobre la adherencia a los tratamientos antirretrovirales. Es claro que la eficacia de las intervenciones sobre adherencia se basa en el desarrollo de estrategias individualizadas, múltiples y repetitivas.

Cuadro 9: estrategias e intervenciones para fomentar la adherencia.

Disponibilidad de tratamientos más simples.

Preparación previa al inicio del tratamiento.

Reuniones específicas de adherencia (individuales o grupos). Adecuación del esquema a las actividades y horarios del paciente.

Involucrar familiares y amigos como promotores de adherencia Control y refuerzo de la adherencia en cada visita al especialista, médico de atención primaria o farmacéutico.

Uso de elementos específicos (calendarios, alarmas, pastilleros).

Bibliografía recomendada
Williams D, Geraci A, Simpson DM. AIDS and AIDS-treatment neuropathies. Curr Neurol Neurosci Rep 2001; 1(6): 553-8.

Bogart LM, Kelly JA, Catz SL y col. Impact of medical and non-medical factors on physician decision making for HIV/AIDS antiretroviral treatment. J Acquir Immune Defic Syndr 2000; 23 : 396-404. Bogart LM, Kelly JA, Catz SL y col. Impact of medical and non-medical factors on physician decision making for HIV/A

Boletín sobre el SIDA en Argentina. Ministerio de Salud de la República Argentina. Año IX № 29, Diciembre de 2012.

Brinkman K, Kakuda TN. Mitochondrial toxicity of nucleoside analogue reverse transcriptase inhibitors: a looming obstacle for long-term antiretroviral therapy? Curr Opin Infect Dis 2000; $13:$
Brinkman K, ter Hofstede HJ. Mitochondrial toxicity of nucleoside analogue reverse transcriptase inhibitors: lactic acidosis, risk factors and therapeutic options. AIDS Rev 1999; 1: 141-8.

Brinkman K, ter Hofstede HJ. Mitochondrial toxicity of nucleoside analogue reverse transcriptase inhibitors: lactic acidosis,

Cahn P, Belloso WH, Murillo J, Prada-Trujillo G. AIDS in Latin America. Infect Dis Clin North
Carr A, Cooper DA. Adverse effects of antiretroviral therapy. Lancet 2000; 356: 1423-30.

Carr A, Cooper DA. Adverse effects of antiretroviral therapy. Lancet 2000; 356: 1423-30.
Clay PG. The abacavir hypersensitivity reaction: a review. Clin Ther 2002; 24(10): 1502-14

Clay PG. The abacavir hypersensitivity reaction: a review. Clin Ther 2002; 24(10): 1502-14.

Daar ES, Cohen C, Remien R y col. Improving adherence to antiretroviral therapy. AIDS Read 2003; 13(2): 81-90.

Daar ES, Little S, Pitt J, et al, Los Angeles County Primary HIV Infection Recruitment Network. Diagnosis of primary HIV-1 infection. Ann Intem Med. 2001;134:25-29.

Dalakas MC. Peripheral neuropathy and antiretroviral drugs. J Peripher Nerv Syst 2001; 6(1): 14-20.

El-Sadr WM, Burman WJ, Grant LB y col. Discontinuation of prophylaxis for Mycobacterium avium complex disease in HIV-infected patients who have a response to antiretroviral therapy, Terry Beim Community Programs for Clinical Research on AIDS. N Engl J Med 2000; 342: 1085-92.

Furrer H, Opravil M, Bemasconi E, y col. Stopping primary prophylaxis in HIV-1 infected patients at high risk of toxoplasma encephalitis. Swiss HIV Cohort Study. Lancet 2000; 355: 2217-8. Gerbert B, Maguire BT, Bleeker Ty col. Primary care physicians and AIDS. Attitudinal and structural barriers to care. JAMA 1991; 266(20): 2837-42.

Giorgi JV, Lyles RH, Matud JL y col. Predictive value of immunologic and virologic markers after long or short duration of HIV-1 infection. J Acquir Immune Defic Syndr 2002; 29(4): 346-55 Goldschmidt RH, Dong BJ. Treatment of AIDS and HIV-related conditions- 2002: Antiretroviral Therapy. J Am Board Fam Prac 2002; 15(4):319-31.

HIV Drug interactions. University of Liverpool. Disponible en: Http://www.HIV-druginteractions.org

Imperiale M. Nevirapine-associated toxicity. AIDS Read 2002 Apr;12(4): 171.

Kasten M. Human Immunodeficiency Virus: The initial physician-patient encounter. Mayo Clin Proc 2002; 77(9): 957-63.

Kaufman GR, Duncombe C, Zaunders J y col. Primary HIV infection: a review of clinical manifestations, immunologic and virologic changes. AIDS Patient Care STDS 1998; 12(10): 759-67.

Kaufman GR, Duncombe C, Zaunders Jy col. Primary HIV infection: a review of clinical manifestations, immunologic and virologic changes. AIDS Patient Care STDS 1998; 12 (10): 759-67.

López Bernaldo de Quirós JC, Miró JM, Pena JM y col. A randomized trial of the discontinuation of primary and secondary prophylaxis against Pneumocystis carinii pneumonia after highly active anti- 
retroviral therapy in patients with HIV infection. Grupo de Estudio del SIDA. N Engl J Med 2001; 344: 159-67.

Masur H, Kaplan J. Current status of opportunistic infections in patients with HIV infection in the era of highly active antiretroviral therapy. Curr Clin Top Infect Dis 2001;21: 64-82 Mellors JW, Muñoz A, Giorgi JV, et al. Plasma viral load and CD4+ lymphocytes as prognostic markers of HIV-1 infection. Ann Intern Med 1997; 126(12): 946-54.

Moore RD, Keruly JC, Chaisson RE. Incidence of pancreatitis in HIV-infected patients receiving nucleoside reverse transcriptase inhibitor drugs.

Moyle G, Back D. Principles and practice of HIV-protease inhibitor pharmacoenhancement. HIV Med 2001; 2(2): 105-13.

Niu MT, Stein DS, Schnittman SM. Primary human immunodeficiency virus type 1 infection: review of pathogenesis and early treatment intervention in humans and animal retrovirus infections. J Infect Niu MT, Stein DS, Schnittman

O'Brien L. Common HIV drug-drug interactions. AIDS Read 1999; 9(2): 104-9.

Orenstein R. Presenting syndromes of Human Immunodeficiency Virus. Mayo Clin Proc 2002; 77(10): 1097-102

Paterson DL, Swindells S, Mohr J y col. Aherence to protease inhibitor therapy and outcomes in patients with HIV infection. Ann Intern Med 2000; 133: 21-30.

Perry CM, Noble S. Didanosine: an updated review of its use in HIV infection. Drugs 1999; 58(6): 1099-135

Phillips KA, Bayer R, Chen JL. New Centers for Diseases Control and Prevention's guidelines on HIV counseling and testing for the general population and pregnant women. J Acquir Immune Defic Syndr 2003; 32(2): 182-91.

Schacker T, Collier AC, Hughes, et al. Clinical and epidemiologic features of primary HIV infection. Ann Intern Med, 1996 125(4): 257-64.

Schambelan M, Benson C, Carr A, Currier JS, Dubé MP, Gerber JG, Grinspoon SK, Grunfeld C, Kotler DP, Mulligan K, Powderly WG, Saag MS. Management of Metabolic complications associated with antiretroviral therapy for HIV-1 infection: Recommendations of an Intemational AIDS Society-USA Panel. J Acquir Immune Defic Syndr 2002; $31: 257-72$

Schrooten W, Dreezen C, Fleerackers Y, Andraghetti R y col. Receiving a positive HIV test result: the experience of patients in Europe. HIV Med 2001; 2(4): 250-4

Selected antiretroviral Drug Interaction Resources. University of California, San Francisco at San Francisco General Hospital. National HIV/AIDS Clinician's Consultation Center. Disponible en: Http://www.ucsf.edu/HIVcntr/Clinical_Resources/Pharmacy/Drug_Intxn Web.htm

Selwyn PA, Amold R. From fate to tragedy: the changing meanings of life, death and AIDS. Ann Intern Med 1998; 129(11): 899-902.

Shapiro MF, Hayward RA, Guillermot D y col. Resident's experience in, and attitudes toward the care of persons with AIDS in Canada, France and the United States. JAMA 1992; 268(4): 510-5.

Stone VE. Strategies for optimizing adherence to highly active antiretroviral therapy: lessons from research and clinical practice. Clin Infect Dis 2001; 33: 865-72.

Tumer BJ, McKee L, Fanning T y col. AIDS specialist versus generalist ambulatory care for advanced HIV infection and impact on hospital use. Med Care 1994; 32(9): 902-16.

Tumer BJ, McKee L, Fanning T y col. AlDS specialist versus generalist ambulatory care for advanced HIV infection
Tumer BJ. Adherence to antiretroviral therapy by HIV-infected patients. J Infect Dis 2002; 185 (suppl 2): S143-51.

Vasa CV, Tang I, Glatt AE. Prophylaxis of opportunistic infections in the HIV-infected patients in the HAART era. Infect Med 2002; 19 (10): $452-60$

Wast CV, Tang I, Glatt AE. Prophylaxis of opportunistic infections in the HIV-infected patients in the HAART era. Infect Med

Watts DH. Management of Human Immunodeficiency virus infection in pregnancy. N.Engl J Med 2002;
May MT, Ingle SM. Life expectancy of HIV-positive adults: a review. Sex Health. 2011 Dec;8(4):526-33.

May MT, Ingle SM. Life expectancy of HIV-positive adults: a review. Sex Health. 2011 Dec;8(4):526-33.

Slavin S, Elliott J, Fairley C, French M, Hoy J, Law M, Lewin S. HIV and aging: an overview of an emerging issue. Sex Health. 2011 Dec;8(4):449-51. Reekie J, Gatell JM, Yust l, Bakowska E, Rakhmanova A, Losso M, Krasnov M, Francioli P, Kowalska JD, Moc

1-positive individuals with high CD4 cell counts according to viral load strata. AIDS. 2011 Nov 28;25(18):2259-68

Petoumenos K, Worm SW. HIV infection, aging and cardiovascular disease: epidemiology and prevention. Sex Health. 2011 Dec;8(4):465-73

Strategies for Management of Antiretroviral Therapy (SMART) Study Group, El-Sadr WM, Lundgren JD, Neaton JD, Gordin F, Abrams D, Arduino RC, Babiker A, Burman W, Clumeck N, Cohen CJ, Cohn D, Cooper D, Darbyshire J, Emery S, Fätkenheuer G, Gazzard B, Grund B, Hoy J, Klingman K, Losso M, Markowitz N, Neuhaus J, Phillips A, Rappoport C. CD4+ count-guided interruption of antiretroviral treatment. N Engl J Med. 2006 Nov 30;355(22):2283-96

Friis-Møller N, Weber R, Reiss P, Thiébaut R, Kirk O, d'Arminio Monforte A, Pradier C, Morfeldt L, Mateu S, Law M, El-Sadr W, De Wit S, Sabin CA, Phillips AN, Lundgren JD; DAD study group. Cardiovascular disease risk factors in HIV patients--association with antiretroviral therapy. Results from the DAD study. AIDS. 2003 May 23;17(8):1179-93.

Weber R, Sabin CA, Friis-Møller N, Reiss P, El-Sadr WM, Kirk O, Dabis F, Law MG, Pradier C, De Wit S, Akerlund B, Calvo G, Monforte A, Rickenbach M, Ledergerber B, Phillips AN, Lundgren JD. Liver-related deaths in persons infected with the human immunodeficiency virus: the D:A:D study. Arch Intem Med. 2006 Aug 14-28;166(15):1632-41.

Smit C, Geskus R, Walker S, Sabin C, Coutinho R, Porter K, Prins M; CASCADE Collaboration. Effective therapy has altered the spectrum of cause-specific mortality following HIV seroconversion. AIDS. 2006 Mar 21;20(5):741-9.

Goulet JL, Fultz SL, Rimland D, Butt A, Gibert C, Rodriguez-Barradas M, Bryant K, Justice AC. Aging and infectious diseases: do patterns of comorbidity vary by HIV status, age, and HIV severity? Clin Infect Dis. 2007 Dec 15:45(12):1593-601.

Belloso WH, Orellana LC, Grinsztejn B, Madero JS, La Rosa A, Veloso VG, Sanchez J, Ismerio Moreira R, Crabtree-Ramirez B, Garcia Messina O, Lasala MB, Peinado J, Losso MH. Analysis of serious non-AIDS events among HIV-infected adults at Latin American sites. HIV Med. 2010 Oct 1;11(9):554-64. Epub 2010 Mar 2

Kitahata MM, Gange SJ, Abraham AG, Meriman B, Saag MS, Justice AC, Hogg RS, Deeks SG, Eron JJ, Brooks JT, Rourke SB, Gill MJ, Bosch RJ, Martin JN, Klein MB, Jacobson LP, Rodriguez B, Sterling TR, Kirk GD, Napravnik S, Rachlis AR, Calzavara LM, Horberg MA, Silverberg MJ, Gebo KA, Goedert JJ, Benson CA, Collier AC, Van Rompaey SE, Crane HM, McKaig RG, Lau B, Freeman Sterling TR, Kirk GD, Napravnik S, Rachlis AR, Calzavara LM, Horberg MA, Silverberg MJ, Gebo KA, Goedert JJ, Benson CA, Collier AC, Van Rompaey SE, Crane HM, McKaig RG, L

AM, Moore RD; NA-ACCORD Investigators. Effect of early versus deferred antiretroviral therapy for HIV on survival. N Engl J Med. 2009 Apr 30;360(18): 1815-26. Epub 2009 Apr 1 , Monforte AD, Saag M, Smith C, Staszewski S, Egger M, Cole SR. Timing of initiation of antiretroviral therapy in AIDS-free HIV-1-infected patients: a collaborative analysis of 18 HIV cohort studies. Lancet. 2009 Apr 18;373(9672):1352-63. Epub 2009 Apr 8. Review

HIV-CAUSAL Collaboration, Cain LE, Logan R, Robins JM, Steme JA, Sabin C, Bansi L, Justice A, Goulet J, van Sighem A, de Wolf F, Bucher HC, von Wyl V, Esteve A, Casabona J, del Amo J, Moreno S, Seng R, Meyer L, Perez-Hoyos S, Muga R, Lodi S, Lanoy E, Costagliola D, Hernan MA. When to initiate combined antiretroviral therapy to reduce mortality and AIDS-defining illness in HIVinfected persons in developed countries: an observational study. Ann Intern Med. 2011 Apr 19;154(8):509-15

Dabis F, Newell ML, Hirschel B. HIV drugs for treatment, and for prevention.

Lancet. 2010 Jun 12;375(9731):2056-7. Epub 2010 May 26.

Rodriguez-Novoa S, Alvarez E, Labarga P, Soriano V. Renal toxicity associated with tenofovir use. Exp Opin Drug Safety 2010; 9(4);545-99

Cooper RD, Wiebe N, Smith N, Keiser P, Naicker S, Tonelli M. Systematic review and meta-analysis: renal safety of tenofovir disoproxil fumarate in HIV-infected patients. Clin Infect Dis 2010; 51(\%):496505 